\title{
Afrique sub-saharienne et hépatite $C$ : défis et perspectives de la mise en œuvre opérationnelle d'outils diagnostiques innovants
}

\section{Sub-Saharan Africa and hepatitis C: challenges and insights regarding the implementation of innovative diagnostics}

Léa Duchesne, Sorbonne Université, INSERM, Institut Pierre Louis d’Epidémiologie et de Santé Publique (UMR-S 1136)

lea.duchesne@iplesp.upmc.fr

Faculté de médecine Saint-Antoine, Bureau 308

27 rue Chaligny

75012 Paris

Victor Duchesne, Centre d'Economie de Paris Nord, UMR 7234

victor.duchesne@univ-paris13.fr

Université Paris 13, UFR SEG, Bureau J301B

99 avenue Jean Baptiste Clément

93430 Villetaneuse

Version acceptée pour publication dans le Journal d'Economie et de Gestion Médicales, volume 36, numéro 5, pp.315-329, en 2018.

La version publiée dans la revue est accessible au lien suivant :

https://www-cairn-info/revue-journal-de-gestion-et-d-economie-medicales-2018-5page-315.htm

Le présent article est mis à disposition conformément à l'article 30 de la loi pour une République numérique.

Duchesne Léa, Duchesne Victor, «Afrique sub-saharienne et hépatite C: défis et perspectives de la mise en œuvre opérationnelle d'outils diagnostiques innovants », Journal de gestion et d'économie médicales, 2018/5 (Vol. 36), p. 315-329. DOI : 10.3917/jgem.185.0315. URL : https://www-cairn-info.ezproxy.univ-paris13.fr/revuejournal-de-gestion-et-d-economie-medicales-2018-5-page-315.htm 


\title{
Résumé
}

Avec l'avènement de nouveaux traitements efficaces chez plus de $90 \%$ des patients, l'élimination de l'hépatite $\mathrm{C}$ chronique est devenue envisageable. Dans les pays en développement, le manque chronique d'infrastructures de diagnostic représente une barrière à leur distribution. La mise au point d'un test virémique utilisable en contexte décentralisé apparait comme un point crucial de la lutte contre l'hépatite C. De nombreuses innovations, reposant sur le principe d'innovations frugales, pourraient permettre de développer un tel test. Malgré leur potentiel, seule une infime partie de ces technologies ont été jusqu'à présent rendues disponibles sur le marché. Les difficultés inhérentes au processus d'innovation dans les pays en développement ainsi que le manque de collaboration entre domaines de recherche, tant au moment de la conception des prototypes (industrialisation, intégration des retours d'expériences) qu'à celui de sa mise sur le marché (homologation, distribution, viabilité), viennent renforcer la difficile mise en place opérationnelle de ces outils diagnostiques innovants.

\begin{abstract}
With the advent of novel treatments with a cure rate higher than $90 \%$, the elimination of chronic hepatitis $\mathrm{C}$ has become achievable. In developing countries, the chronic lack of diagnostic facilities represents a barrier to their spread. The development of a viremic test suitable to decentralized settings appears to be a crucial point of the fight against hepatitis C. Many innovations, based on the principle of frugal innovations, could enable the creation of such a test. Despite their potential, only a small number of these technologies have been made commercially available. The inherent difficulties of the innovation process in developing countries, as well as the lack of collaboration between the different research fields involved during the design phase of the prototypes (manufacturing, integration of feedbacks) and the marketing phase (regulation, distribution, sustainability), reinforce the difficulty of implementing innovative diagnostics.
\end{abstract}

Mots-clés : Hépatite C, diagnostic, Afrique, Innovations frugales,

Keywords: Hepatitis C, diagnostic, Africa, frugal innovations 


\section{Introduction}

Les avancées thérapeutiques et sanitaires des vingt dernières années, accompagnées d'une plus grande disponibilité des médicaments ainsi que d'une amélioration des services de santé dans le monde et en particulier dans les pays en de développement (PED) et les moins avancés (PMA), ont conduit à une diminution du poids des maladies transmissibles dans la mortalité mondiale au profit des maladies non-transmissibles et chroniques [1]. Géographiquement, une des exceptions à ce phénomène est l'Afrique sub-saharienne où les maladies transmissibles restent parmi les premières causes de mortalité. Autre exception, les hépatites virales qui, contrairement à la majorité des maladies virales, ont vu leur taux de mortalité augmenter ces dix dernières années [1]. Il a été estimé qu'en 2015, 1,34 million de décès pouvaient leurs être imputées, les plaçant ainsi au même niveau que le paludisme, la tuberculose ou le VIH dans le classement des causes de mortalité dans le monde.

Cinq principaux virus de l'hépatite, désignés par les lettres A, B, C, D ou delta et E, ont été à ce jour identifiés, les deux plus répandus étant les virus de l'hépatite $B$ et $C$. Bien que la prévalence, c'est-à-dire la proportion de personnes touchées par une maladie, de l'hépatite $B$ soit bien plus élevée que celle de l'hépatite $C$ avec 257 millions de personnes infectées par le VHB contre 71 millions pour le VHC, la progression de l'épidémie du VHC est bien plus active que celle du VHB [2]. Ainsi, on estime qu'en 2015 il y a eu plus d'1,75 million de nouveaux cas d'infection par le VHC [2]. Contrairement au VHB, aucun vaccin contre le VHC n'est actuellement disponible. La lutte contre l'hépatite $C$ repose donc uniquement sur le traitement thérapeutique des personnes infectées identifiées et la prévention de la transmission. Or, pendant des années, le seul traitement de l'hépatite C disponible reposait sur l'injection hebdomadaire d'Interféron pegylé, parfois pendant plus d'un an, et ce avec un taux de guérison d’à peine 50\%, voire moins chez les personnes présentant un stade avancé de la maladie hépatique. De plus, ce traitement présentait des effets secondaires forts entrainant de nombreuses interruptions de traitement. Le suivi du patient requis par un tel traitement était donc complexe et quasiment impossible à mettre en place dans des pays à faibles ressources en santé.

En 2011, avec la mise sur le marché des premiers antiviraux à action directe (AAD), plus efficaces et mieux tolérés que le traitement à base d'interféron, le paradigme de prise en charge de l'hépatite $\mathrm{C}$ a radicalement changé. En effet, les AAD se présentent sous forme orale, requièrent une durée moyenne de traitement de 3 mois, présentent un taux de guérison d'environ $90 \%$ chez tous les types de patients et n'ont presque aucun effet secondaire. Ces traitements ont ainsi permis l'allègement, d'une part, des restrictions de mise sous traitement dont l'accès a pu être ouvert à des patients autrefois considérés comme difficiles à traiter et, d'autre part, du suivi des patients, rendant ainsi envisageable la mise en œuvre de programmes de soins du VHC dans des contextes de ressources en santé restreintes. En 2016, 1,76 million de personnes ont été mises sous traitement, faisant passer la couverture thérapeutique des personnes diagnostiquées de 7 
à 13\% [3]. Bien que positive, cette augmentation reste encore trop faible pour permettre d'éliminer l'hépatite $\mathrm{C}$ chronique. Ce décalage entre le potentiel des AAD et leur faible mise en œuvre résulte tout d'abord de leur prix qui était jusqu'à encore récemment trop élevé pour les PED-PMA où vivent la majorité des personnes infectées par le VHC, mais aussi du manque de moyens dédiés au reste de la cascade de soins : le dépistage, le diagnostic et le suivi des patients. Il a été estimé que seules $20 \%$ des personnes infectées dans le monde ont été à ce jour diagnostiquées [2]. Dans les PED-PMA, où vivent 75\% des personnes touchées par le VHC [4], ce taux tombe à 5\%.

Ce taux extrêmement bas résulte d'un ensemble d'obstacles techniques, économiques et organisationnels qui empêchent actuellement la mise en place de structures de diagnostic de l'hépatite $\mathrm{C}$ chronique dans les pays du Sud. Cet article a pour objectif de décrire ces barrières ainsi que d'identifier certains points de levier qui pourraient permettre de les dépasser. Cet article présentera dans une première partie les enjeux liés à une meilleure prise en compte de la phase de diagnostic dans la lutte contre le VHC, les solutions techniques actuellement mises en place pour y faire face ainsi que leurs limites. Dans une deuxième partie nous montrerons en quoi certaines innovations pourraient entrainer un vrai changement de paradigme dans le développement de tests diagnostic délocalisés et peuvent correspondre à un processus d'innovation pensé en termes d'innovations frugales. Enfin dans une troisième partie, nous sortirons du laboratoire pour identifier des pistes de réflexion permettant de faciliter la mise en œuvre et la diffusion de ces outils diagnostics innovants sur le terrain.

\section{L'hépatite C au Sud}

\subsection{Prise en charge de l'hépatite $C$ en 2016 : opportunités et limites de la révolution des Antiviraux à action directe}

Les AAD, par leur simplicité d'administration et leur faible durée de traitement, offrent en théorie le moyen d'étendre pleinement la distribution à grande échelle du traitement de l'hépatite C. Leur prix, au départ trop élevé pour pouvoir permettre leur adoption par les PED-PMA, ont considérablement baissé depuis 2015, principalement grâce à l'émission de licences volontaires et au développement de versions génériques. Il est ainsi estimé que $60 \%$ des personnes infectées par le VHC vivent dans des pays où les AAD sont théoriquement accessibles [5]. Cependant, encore peu de pays ont développé des programmes d'accès à ces molécules, principalement à cause de leur manque d'infrastructures de diagnostic et de soins, nécessaires au suivi de qualité des patients.

Avec les premières combinaisons d'AAD, la mise sous traitement requiert un bilan préthérapeutique complet incluant une mesure de l'avancement de la maladie hépatique du patient ainsi que la détermination du génotype du virus, ce dernier pouvant influencer l'efficacité de certaines molécules. Les dernières générations d’AAD, dites pangénotypiques car pouvant être administrées à tout patient sans distinction de génotype du VHC, permettent de se contenter d'un bilan pré-thérapeutique n’incluant 
que le diagnostic du VHC. La mesure du niveau de fibrose hépatique reste nécessaire pour un suivi sur le long terme mais n’influence en rien la prescription thérapeutique. Cependant, l'accès à ces molécules dans les PED-PMA est encore très restreint.

Les multiples stratégies adoptées pour faire baisser les prix des premières combinaisons d'AAD (licences obligatoires, génériques, tarification différenciée, programme d'accès des industriels [6]) ainsi que l'implication grandissante des instances internationales et nationales devrait permettre de faire baisser le prix des traitements pangénotypiques. Une fois ces molécules rendues disponibles, le manque d'infrastructures de soins constituera donc le principal obstacle de l'accès au soin du VHC dans les PED-PMA.

\subsection{Dépasser le médicament : les enjeux du diagnostic au Sud}

Le dépistage et le diagnostic des personnes infectées par le VHC constituent la première marche de la cascade de soins de l'hépatite $C$ chronique. Le faible taux actuel de diagnostic du VHC représente donc l'un des principaux manques en termes d'infrastructures de soins de l'hépatite C.

Le diagnostic des personnes infectées par le VHC repose sur la détection de la molécule d'ARN (acide ribonucléique) ${ }^{1}$ du VHC, la présence de ce dernier indiquant une activité de réplication de la part du virus et donc une infection dite "chronique ». Or, la détection d'ARN étant une technique onéreuse et complexe, la procédure de référence préconise d'identifier au préalable les personnes ayant déjà été en contact avec le VHC en utilisant un premier test moins coûteux, la recherche des anticorps anti-VHC, ces derniers étant produits par toute personne ayant été infectée par le VHC. Cependant, $30 \%$ des personnes infectées éliminent naturellement le virus et ne deviennent pas porteurs chroniques. La recherche d'ARN reste donc nécessaire chez les personnes ayant obtenu un résultat positif pour le test d'anticorps afin de confirmer le diagnostic. La prévalence de l'hépatite $C$ en population générale étant souvent faible, cette procédure en deux étapes permet d'éviter les coûts inutiles qu'engendrerait la réalisation d'un examen virémique chez ces personnes non chroniques. Cependant, cette procédure représente en elle-même un obstacle à la mise en place d'une stratégie de diagnostic à grande échelle dans les PED-PMA.

En effet, ces outils virologiques, utilisés en routine dans les pays développés, sont souvent inexistants ou coûteux dans les PED-PMA. Les laboratoires possédant à la fois les plateformes d’analyse nécessaires à leur exécution, des appareils encombrants, coûteux et nécessitant une maintenance régulière, ainsi que le personnel qualifié pouvant les utiliser sont rares et restreints aux grandes villes. Dans des pays où plus de

\footnotetext{
1 L'acide ribonucléique, tout comme l'ADN (acide désoxyribonucléique), est une molécule contenant les informations génétiques héréditaires d'un organisme. Dans le cas du virus de l'hépatite $C$, c'est une molécule d'ARN qui détient le génome du virus. Une fois dans l'organisme de la personne infectée, le VHC va se fixer sur les cellules de son foie afin d'y introduire son ARN. En utilisant la machinerie cellulaire de ces cellules, le virus va alors pouvoir répliquer son matériel génétique et ainsi se multiplier.
} 
la moitié de la population vit en zone rurale, cette centralisation des moyens réduit considérablement les possibilités d'accès au diagnostic des populations [7]. Le transport des échantillons depuis les zones rurales jusqu'aux laboratoires centraux est par ailleurs difficilement envisageable. En effet, ces techniques requièrent un volume de sang conséquent qui doit donc être prélevé par ponction veineuse. Or ce type d'échantillon doit être conservé à basse température afin d'éviter la dégradation des biomarqueurs. Leur transport nécessiterait donc la mise en œuvre d'un circuit de transport réfrigéré. Au vu de l'état des infrastructures routières dans les PED-PMA et du coût qu'engendrerait la mise en œuvre d'un tel circuit, ce type de solution n’a encore jamais été privilégié. Il revient donc au patient de se déplacer jusqu'au centre de prélèvement, malgré les coûts que cela peut engendrer. Lorsque les capacités diagnostiques des laboratoires centraux sont trop faibles pour absorber la demande, ces institutions ont recours à l'externalisation des analyses virologiques vers des pays mieux équipés, par exemple de l'Afrique de l'Ouest vers l'Europe.

Ce phénomène entraine une augmentation considérable du prix des tests diagnostiques, déjà élevé lorsqu'ils sont effectués sur place. Dans les PED-PMA, un test de charge virale dépasse souvent le salaire minimum de ces pays, voire le PIB par habitant, rendant inenvisageable le recours à ce service. Le coût des tests de charge virale ainsi que les délais très longs de réponse entre les deux tests, dus au manque d'infrastructure, entrainent la sortie du circuit de diagnostic d'un nombre important de patients dépistés positifs, en particulier chez les populations ayant des difficultés à atteindre les infrastructures de diagnostic.

A ces limites techniques s'ajoutent des limites organisationnelles liées à la structure des systèmes de santé des PED-PMA aggravées par le manque d’investissement dans la lutte contre l’hépatite $\mathrm{C}$ qui a caractérisé les politiques en santé de ces pays depuis des années.

Tout d'abord, le manque de lien entre les infrastructures de diagnostic et celles de soins entraine une faible rétention dans le système de soin des personnes, déjà rares, allant jusqu'au bout de la procédure diagnostique. Dans le cas précis de l’hépatite $C$, la faible disponibilité des traitements dans ces pays a aussi freiné les recommandations de dépistage par les autorités de santé, aucune solution thérapeutique ne pouvant être proposée à l'issue du diagnostic.

Par ailleurs, le manque de personnel de santé, en particulier dans les zones excentrées, est un frein systémique à l'accès aux soins dans les PED-PMA [8]. A fortiori, les spécialistes à même de prendre en charge les hépatites virales (hépatologues et infectiologues) sont peu nombreux et concentrés eux aussi dans les centres de référence des grandes villes. Afin de pallier ce manque, de nombreux circuits alternatifs de prise en charge des patients, reposant sur les médecins généralistes et les travailleurs sociaux, ont été créés dans le but de décentraliser leur prise en charge [9]. Cependant, l’hépatite $\mathrm{C}$ ayant été une maladie négligée jusqu’à l'arrivée des $\mathrm{AAD}$, peu de PED-PMA 
disposent à ce jour de programme de prise en charge et de prévention de l'hépatite $C$ permettant d'organiser ce genre de système [10]. Les connaissances de l'hépatite C chez les populations de ces pays et les professionnels de santé restent parcellaires [11], freinant le recours au diagnostic du VHC ou sa prescription. Enfin, l'absence de fonds, nationaux ou internationaux comme il peut en exister pour le VIH ou la tuberculose, restreint la mise en place de tels programmes ou de subvention des soins, laissant le coût du diagnostic à la charge du patient.

\subsection{Outils diagnostiques alternatifs actuellement disponibles : état des lieux et limites}

La réduction des coûts des tests virologiques, en particulier celui confirmant la virémie, ainsi que l'adoption de systèmes permettant aux populations marginalisées ou excentrées d'avoir accès au diagnostic, sont donc primordiaux pour étendre l'accès aux soins de l’hépatite C. Plusieurs outils ont été développés dans ce but.

Dans le cas du VHC, le test virémique de référence repose sur la détection de son ARN. Cependant, il a été démontré que la détection de l'ARN pouvait être remplacée par celle d'un autre marqueur de la réplication virale, l'antigène core du VHC [12], plus simple et moins cher à détecter que l'ARN du VHC. Cependant, les seules plateformes permettant sa détection disponibles actuellement ne peuvent être utilisées que dans le cadre d'un laboratoire de virologie, avec les mêmes exigences que les techniques traditionnelles de détection de l'ARN du VHC. Son utilisation permettrait donc à la fois la baisse du coût de la procédure diagnostique et sa simplification mais ne réglerait pas le problème de la centralisation des capacités diagnostiques.

Le prélèvement sur papier buvard, aussi appelé «dried blood spot » (DBS), a été développé comme une alternative aux techniques de prélèvement traditionnelles dont le caractère invasif pouvait être délétère chez certaines populations telles que les nourrissons ou les usagers de drogue. Cette technique repose sur le dépôt d'une goutte de sang capillaire prélevé au bout du doigt sur un papier buvard spécifique qui est ensuite séché. Contrairement aux échantillons sanguins classiques qui doivent être congelés pour être transportés, les DBS peuvent être conservés à température ambiante pendant plusieurs mois. De récentes études ont montré que les performances diagnostiques des techniques de détection des anticorps anti-VHC et de l'ARN VHC sur DBS étaient similaires à celles sur échantillons traditionnels [13]. En revanche, bien que les estimations varient en fonction des études, la détection de l'antigène du core du VHC sur DBS semble moins bonne que sur échantillons traditionnels [13] [14]. Couplée à une baisse des prix des tests, l'utilisation de DBS pourrait permettre d'étendre l'accès au diagnostic et de réduire le nombre de perdus de vue entre les deux étapes de la procédure diagnostique.

Les tests rapides, dont l'un des exemples le plus connu est le test de grossesse vendu en pharmacie, permettent d'effectuer une détection de marqueur virologique en contexte 
délocalisé, que ce soit dans un centre de santé de proximité ou au chevet même du patient, d'où leur dénomination anglaise de tests "point of care » (POC). L’Organisation Mondiale de la Santé (OMS) a développé une liste de critères que devraient respecter les tests POC afin d'être en adéquation avec les besoins du terrain. Ces tests devraient être peu coûteux, précis, simples à utiliser et à interpréter, rapides, stables dans des conditions extrêmes, ne nécessiter aucun ou peu de matériel ancillaire et être culturellement acceptables. De tels tests existent depuis maintenant des années pour la détection des anticorps anti-VHC mais leur technologie n'a été que récemment appliquée à la détection de l'ARN du VHC. A l'heure actuelle, le test GeneXpert est le seul à être disponible sur le marché. Ce test se présente sous la forme d'une cartouche contenant l'échantillon de sang et être insérée dans une plateforme qui effectue l'analyse de manière automatisée. Ce système fermé permet d'éviter la contamination de l'échantillon et ne nécessite qu'un entrainement minimal des agents devant l'utiliser. L'autre avantage est que cette machine est déjà utilisée dans certains PED-PMA dans le cadre du diagnostic d'autres maladies, en particulier la tuberculose. Avec des performances quasiment similaires à celles des techniques de laboratoire [15], un temps de réponse de 30 minutes et sa facilité d'utilisation, le test GeneXpert respecte la majeure partie des critères de cette liste. Cependant, son besoin d'un apport continu en électricité et sa taille avoisinant celle d'un four micro-onde restreignent sa mise en œuvre aux centres de référence ou de proximité, encore trop centralisés pour atteindre les populations excentrées.

Ces outils représentent un premier pas vers la simplification ainsi que la décentralisation du diagnostic dans les PED-PMA. Cependant, les limites découlant de leurs propriétés intrinsèques rendent nécessaire le développement d'innovations plus poussées afin d'atteindre pleinement cet objectif.

\section{Quelles innovations diagnostiques?}

\section{1. « Laboratoire sur puce » : solutions en cours de développement}

La détection de matériel génétique, pierre angulaire de la majorité des procédures diagnostiques de maladies infectieuses, repose sur un ensemble complexe d'étapes. Le matériel génétique est d'abord isolé du reste des composants de la matrice qui a été recueillie (sang, salive, mucus) afin de retirer toute source possible de contamination qui pourrait fausser la détection. Ensuite, le matériel génétique étant présent en infime quantité dans ce genre d'échantillon, le marqueur ciblé par le test doit être amplifié. Cette technique requiert d'exposer l'échantillon à des températures variables selon un rythme précis et nécessite donc un équipement d'émission et de régulation de chaleur complexe. Enfin, le marqueur amplifié est détecté, le plus souvent via la mesure d’un signal émis par une molécule-traceur dont l'affinité naturelle avec le marqueur cible aura permis de les lier au cours du processus. Ce signal doit alors être transmis sous une forme interprétable par l'agent effectuant le test via une interface. Intégrer toutes ces 
étapes dans un seul dispositif miniature, automatisé, à bas coût et répondant aux critères d’efficacité de l’OMS représente un vrai défi technique.

La course à la miniaturisation que connait l'industrie depuis plusieurs décennies a conduit au développement d'innovations permettant la réplication d'une grande variété de techniques industrielles ou de laboratoire à l'échelle micro voire nanoscopique. Grâce à la collaboration de divers champs de recherche, allant de la biologie, la chimie au développement de logiciel ou la science des matériaux, cette tendance a permis l'émergence du concept de "laboratoire sur puce " (laboratory on a chip) visant à réunir en un seul dispositif miniature plusieurs processus de laboratoire [16].

L'un des piliers de ce développement est la science des microfluides qui permet de manipuler de très faibles volumes de liquides grâce aux propriétés physiques de microconduits. Développée pour le domaine de l'électronique avec la création de puces en polymères, cette technologie a pu être transposée à la mise au point de laboratoires sur puce à visée diagnostique. Ainsi, des dispositifs miniatures pré-chargés en réactifs ne nécessitant que l'injection d'un échantillon liquide pour déclencher une suite d'opérations (séparation, mélange, vortex, etc.) selon une séquence prédéfinie par la forme même du dispositif ont pu être créés. La plupart de ces dispositifs nécessitent encore l'ajout externe d'une pompe permettant d'injecter l'échantillon, ce qui les rend moins simples d'utilisation, mais des solutions sont en cours de développement afin d'automatiser complètement leur fonctionnement et de rendre leur utilisation accessible à tous. Le développement des imprimantes 3D a rendu possible l'insertion de cette technologie dans des supports bon marché, compatibles avec les réactifs utilisés lors des analyses biologiques et pouvant résister aux contextes difficiles d'utilisation des tests POC (papier, thermoplastiques) [17] [18].

Pour le processus de détection même, des techniques d'amplification isothermes ont été développées afin de réduire au minimum les besoins en équipement externe de production et de contrôle de chaleur. Développés au départ par des biologistes et des chimistes pour simplifier les appareils de laboratoire, ces techniques trouvent maintenant leur place dans le développement de tests POC. Cependant, ces techniques étant brevetées, le coût des licences d'exploitation pourrait empêcher le développement de dispositifs à un prix abordable pour les PED-PMA. D'autres systèmes de détection ne reposant pas sur l'amplification du matériel génétique sont en cours de développement et ont montré un potentiel intéressant pour leur intégration dans des laboratoires sur puce [19] [20].

Dans le but de pouvoir être utilisé par des personnes avec un minimum d'entrainement, le résultat des tests POC doit être facilement interprétable. Les tests qualitatifs, c'est-àdire avec un résultat binaire, peuvent ne donner qu'une réponse colorimétrique, à l'instar des tests de grossesse. Les tests quantitatifs posent un problème plus complexe et requièrent souvent le recours à une technologie avancée permettant la traduction du signal émis par le test en une réponse chiffrée. Au vue de la croissance qu’a connue le 
marché de la téléphonie mobile dans les PED-PMA, de nombreux laboratoires de recherche ont cherché à inclure leur utilisation dans le processus de délocalisation du diagnostic, à travers des procédures plus ou moins complexes [21] [22]. L'une des solutions les plus simples proposées consiste en la mise au point d'un test colorimétrique dont le résultat serait pris en photo par l'agent de santé puis envoyé à un technicien se trouvant dans un laboratoire central possédant des logiciels d'analyse d'image capables de déduire de la photo un résultat quantitatif. Sur le même principe, l'utilisation d'un logiciel d'analyse stocké sur le réseau internet, paramétré pour un fonctionnement automatisé et distant, a montré de bons résultats [23]. Enfin, le portable en lui-même pourrait servir de logiciel d'analyse, soit en l'équipant d'un logiciel compatible avec ce type d'appareil soit en servant de base pour un appareil annexe miniaturisé d'analyse.

Au-delà du développement d'outils miniaturisés, certains groupes de recherche ont aussi pour but de limiter le coût de fabrication de tels dispositifs. Ainsi, Pardee et son équipe ont développé un test colorimétrique de détection de l'ARN du Zika sur papier pour un coût de développement de 20\$ par prototype et un coût final par test oscillant entre 0,1 et $1 \$$ [24]. Cependant, la sensibilité de ce test est encore trop faible pour qu'il soit utilisé sans effectuer une étape d'amplification au préalable et nécessite donc de plus amples développements pour être opérationnel dans un contexte délocalisé. D’autres se sont appuyés sur des objets du quotidien afin de développer des dispositifs simples d'utilisation et à bas coût, tels qu'un batteur à œuf [25], une essoreuse à salade [26] ou une toupie d'enfant [27]. La plupart des innovations citées plus haut ont été développées afin de résoudre un problème donné dans une situation donnée. Certains groupes de recherche ont avancé l'hypothèse que la mise au point de laboratoires sur puces reprogrammables pourrait être une solution afin de réduire les coûts et la durée de la phase de développement. Une équipe a ainsi construit une structure de laboratoire sur puce sur une base de boîte à musique [28]. Un système de carte perforée permet de déclencher selon les besoins l'ouverture des valves ou d'actionner les pompes de la puce pré-chargée en réactifs. Le système de manivelle et de picots de la boîte à musique permet de lire la carte sans apport électrique. La carte ainsi que la puce sont faites de matériaux peu coûteux (moins de 5\$), peuvent être modifiées en fonction des besoins de l'utilisateur, permettant ainsi une grande variété de combinaisons d'action, et peuvent être réutilisées une fois lavées.

\subsection{Innovations frugales ou théoriser le « système $D$ "}

La lutte contre l'Hépatite $\mathrm{C}$ en Afrique, de son diagnostic à son traitement, doit faire face à deux contraintes majeures. Une première liée au contexte particulier dans lequel elle se développe, à savoir le contexte économique et social des PED-PMA africains. Une seconde liée à la complexité même des différentes étapes de mise œuvre de la prise en charge des personnes atteintes de l'hépatite C ( $c f$. section 1). Bien que ces deux 
contraintes ne sont pas en soi spécifiques à l'hépatite $\mathrm{C}$ et concernent plus généralement l'ensemble des maladies infectieuses en Afrique, l'hépatite C par son ampleur et sa nouveauté apparait comme un exemple significatif de ce contexte particulier. Dans ces conditions, les industriels pharmaceutiques, les professionnels et les acteurs institutionnels de santé ont donc été amenés à développer de nouveaux dispositifs tentant de répondre à ces contraintes concernant notamment la première étape de la prise en charge de l'hépatite $C$ : le diagnostic ( $c f$. section 2.1). Pour saisir la portée et l’originalité de ces différents dispositifs de diagnostic de l'hépatite $C$, il est nécessaire de dépasser les schémas traditionnels d'analyse et de s’intéresser aux développements récents en particulier en matière d'économie de l'innovation.

En effet, l'analyse des questions de santé au Sud est traditionnellement menée dans une perspective macroéconomique soit en termes de relation Nord-Sud (transferts, nouveaux marchés) soit à travers l'analyse des complexes pharmaco-industriels nationaux (modèle productif, système national d'innovation). En matière d'innovation, les problématiques sont principalement posées dans une perspective de propriété intellectuelle (droits, brevets). Le contexte particulier et la nature même des nouveaux dispositifs de diagnostic obligent les chercheurs à renverser leur perspective d'analyse en passant d'un point de vue macroéconomique à un point de vue microéconomique centré directement sur le producteur de soins se trouvant au plus proche du terrain. Il ne s'agit plus de caractériser un modèle productif ou un régime d'innovation national existant ou souhaitable mais de rendre compte de la manière dont les différents acteurs, ici en santé, dépassent les contraintes qui s'imposent à eux en recombinant les ressources à leur disposition afin de répondre aux besoins de santé d’une population [29].

Dans leur revue de la littérature consacrée aux conceptualisations récentes sur les innovations en santé au Sud, Haudeville et Le Bas caractérisent 5 types d'innovations

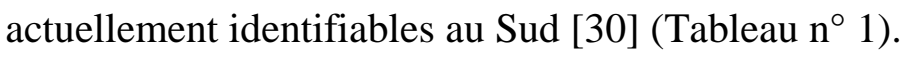


Tableau $\mathbf{n}^{\circ}$ 1. Différents concepts d'innovation : définition et impacts [30]

\begin{tabular}{|c|c|c|c|c|}
\hline $\begin{array}{c}\text { Types } \\
\text { d'innovations }\end{array}$ & Définition & $\begin{array}{c}\text { Impacts } \\
\text { économiques } \\
\text { généraux }\end{array}$ & $\begin{array}{c}\text { Implications } \\
\text { stratégiques } \\
\text { pour les firmes }\end{array}$ & Références \\
\hline $\begin{array}{c}\text { Innovation } \\
\text { frugale }\end{array}$ & $\begin{array}{l}\text { Nouvelle } \\
\text { conception des } \\
\text { produits }\end{array}$ & $\begin{array}{l}\text { Réduction des } \\
\text { coûts } \\
\text { + répond aux } \\
\text { besoins du } \\
\text { Bottom Of the } \\
\text { Pyramid }\end{array}$ & $\begin{array}{c}\text { Nouveaux } \\
\text { segments de } \\
\text { marché } \\
\text { desservis }\end{array}$ & $\begin{array}{l}\text { Basu et al. } \\
\qquad \begin{array}{c}\text { (2013) } \\
\text { Radjou et } \\
\text { al. } \\
(2013)\end{array}\end{array}$ \\
\hline $\begin{array}{l}\text { Low-cost } \\
\text { innovation }\end{array}$ & $\begin{array}{c}\text { Même } \\
\text { fonctionnalité que } \\
\text { les produits du } \\
\text { Nord mais coûts } \\
\text { moindres }\end{array}$ & $\begin{array}{c}\text { Minimisation } \\
\text { des } \\
\text { coûts par baisse } \\
\text { du nombre de } \\
\text { fonctionnalités }\end{array}$ & $\begin{array}{l}\text { Attaque de } \\
\text { nouveaux } \\
\text { segments de } \\
\text { clientèle }\end{array}$ & $\begin{array}{l}\text { Zeschkyet } \\
\text { al. } \\
\text { (2014) }\end{array}$ \\
\hline $\begin{array}{c}\text { Good-enough } \\
\text { innovation }\end{array}$ & $\begin{array}{c}\text { Nouvelles } \\
\text { fonctionnalités }\end{array}$ & $\begin{array}{c}\text { Bonnes } \\
\text { correspondances } \\
\text { avec la demande } \\
\text { des } \\
\text { consommateurs }\end{array}$ & $\begin{array}{c}\text { Similaire à la } \\
\text { low cost } \\
\text { innovation }\end{array}$ & $\begin{array}{c}\text { Zeschkyet } \\
\text { al. } \\
\text { (2014) }\end{array}$ \\
\hline $\begin{array}{l}\text { Innovation } \\
\text { inverse }\end{array}$ & $\begin{array}{c}\text { Transfert des idées } \\
\text { du Sud vers le } \\
\text { Nord }\end{array}$ & $\begin{array}{c}\text { Ce sont les } \\
\text { marchés } \\
\text { qui priment et } \\
\text { non la } \\
\text { technologie }\end{array}$ & $\begin{array}{l}\text { Au cœur des } \\
\text { stratégies des } \\
\text { FMN du Sud } \\
\text { comme du } \\
\text { Nord (en } \\
\text { concurrence) }\end{array}$ & $\begin{array}{l}\text { Bremet } \\
\text { Ivens } \\
\text { (2013) }\end{array}$ \\
\hline $\begin{array}{l}\text { Innovation } \\
\text { durable }\end{array}$ & $\begin{array}{l}\text { Mix d'innovations } \\
\text { environnementales } \\
\text { et sociales }\end{array}$ & $\begin{array}{l}\text { Participe de } \\
\text { l’économie } \\
\text { circulaire }\end{array}$ & $\begin{array}{l}\text { Les firmes se } \\
\text { positionnent } \\
\text { sur les } \\
\text { produits vers et } \\
\text { la RSE }\end{array}$ & $\begin{array}{c}\text { Ketata et al. } \\
\text { (2014) }\end{array}$ \\
\hline
\end{tabular}


Parmi ces différentes catégories, ils retiennent particulièrement celle d'innovation frugale car elle apparait comme la plus complète dans la mesure où elle englobe en partie les autres conceptions de l'innovation évoquées. Le concept d'innovation frugale a majoritairement fait l'objet de développement dans la littérature et les pays anglophones, notamment en Inde. Il reste peu développé dans le cadre francophone [31]. Plus généralement, il reste réservé aux pays du Sud même si certaines analyses tentent de l'appliquer aux pays du Nord comme la Grande-Bretagne [32], renversant ainsi la relation Nord-Sud. L'innovation frugale peut être définie comme la recombinaison de savoirs existants à travers un dispositif (produit ou service) caractérisé par sa simplicité, c'est-à-dire une faible intensité technologique et un faible coût (financier, institutionnel) afin de répondre à un besoin non encore satisfait tout en garantissant un haut degré de qualité pour son utilisateur.

Parce qu'il semble être en mesure de répondre à de nombreuse contraintes économiques et sociales actuelles, le concept d'innovation frugale offre donc un cadre conceptuel séduisant tant pour les décideurs que pour les chercheurs pour saisir et promouvoir les innovations en santé au Sud. Cependant, il ne faut pas faire de l'innovation frugale un concept miracle car de nombreuses contraintes demeurent. En effet, pour qu'une innovation soit en mesure de répondre potentiellement à un besoin de santé non satisfait, encore faut-il qu'elle ait été diffusée, c'est-à-dire sortie d'un cadre expérimental ou de laboratoire. C'est là l'enjeu actuel des innovations frugales : réussir à être diffusées largement [33].

\section{Du laboratoire au terrain, quels obstacles?}

\subsection{De la conception...}

Malgré l’intérêt que présentent les dispositifs de laboratoires sur puces pour dépasser les limites actuelles des tests POC, peu de prototypes ont dépassé l'étape de la preuve de concept et été produits puis adoptés sur le terrain.

Bien que les difficultés rencontrées lors de la conception de tests POC nécessitent de faire appel à des champs de recherche très variés qui ne sont pas forcément naturellement connectés, des synergies avec le domaine biomédical se sont développées, comme le montrent les exemples de la section 2.1. Cependant, le cloisonnement entre champs de recherche reste fort. Sackmann et son équipe ont ainsi montré que les articles portant sur les systèmes microfluidiques étaient principalement publiés dans des journaux d'ingénierie [34], rendant leur accès limité aux cliniciens et biologistes qui font pourtant partie des principaux potentiels usagers de ces technologies. Afin de garantir l'adoption future de ces dispositifs dans la pratique clinique, il parait nécessaire de rendre plus accessible la connaissance de ce genre de dispositifs aux laboratoires et cliniciens, voire même de les inclure dans le processus de création. Par ailleurs, une telle collaboration pourrait garantir l'adéquation entre les caractéristiques des prototypes développés et les besoins du terrain, de nombreux prototypes étant encore trop centrés 
sur la résolution d'un problème technique spécifique sans être inclus dans une vision globale du produit final à fournir. Ce décalage a aussi des répercussions sur les possibilités d'industrialisation de ces dispositifs.

En effet, le coût des processus de fabrication choisis pour une production à petite échelle en laboratoire, sans procédure de contrôle de qualité ou de finition, peut s'avérer bien plus élevé lors du passage à une production à grande échelle. Cependant, peu de bourses de recherche fondamentale valorisent l'étude de ces aspects pratiques. Par conséquent, peu de prototypes développés dans le cadre de la recherche académique prennent en compte les exigences d'une production industrielle [35]. Une plus forte collaboration entre les différents acteurs de la chaîne de développement pourrait aussi élargir les possibilités de financement de ce genre de recherche.

\section{2. ... à la viabilité économique et au cadre réglementaire}

Bien que les besoins en outils diagnostiques soient considérables dans les PED-PMA, le marché des tests diagnostiques y est considéré comme à risque et attire peu d'investisseurs. En effet, alors que le prix des tests POC doit rester bas, leur développement requiert un investissement conséquent.

Dans de nombreux pays, les outils de diagnostic in vitro doivent passer par un processus d'homologation avant de pouvoir être commercialisés. Bien que ces procédures aient pour but de garantir la sécurité des populations, elles sont souvent mal définies et variables en fonction des pays. Dans la plupart des cas, il est demandé aux fabricants de conduire un essai afin de prouver l'efficacité du test dans les futures conditions d'utilisation. De tels essais demandent un investissement conséquent ainsi que d'avoir accès aux structures locales. Le manque d'harmonisation entre les procédures des pays, le coût et la répétition nécessaire de ces procédures conduisent les fabricants à limiter le nombre de pays dans lesquels ils commercialisent leur produit, participant ainsi à l'accès limité aux outils diagnostiques dans les PED-PMA.

Des initiatives ont été lancées à différentes échelles géographiques afin d’harmoniser ces procédures, comme l'International Medical Device Regulators Forum et les regional Harmonization Working Parties d'Afrique, Amérique Latine et Asie (36). Des organismes semblables créés pour harmoniser les procédures d’homologation des médicaments, comme le système harmonisé d'enregistrement des médicaments établi par la Communauté de l'Afrique de l'Est (EAC) [37], ont montré des résultats prometteurs.

\subsection{Dépasser les contraintes : libre accès et coopération}

L'accès aux outils diagnostiques repose sur la disponibilité d'outils adaptés mais aussi sur le système économique et commercial qui régit leur distribution. Repenser ce système afin d'en dépasser les limites pourrait aussi permettre d'élargir cet accès 
comme le suggèrent notamment les le projet OPP-ERA (Open Polyvalent Platform Era) et les stratégies reposant sur le libre accès (open source).

Le projet OPP-ERA, mis en place en 2013 dans quatre pays d'Afrique sub-saharienne par un consortium d'organisations non gouvernementales (UNITAID, Solthis, Sidaction) et d'institutions de recherche publiques (Agence National de recherche sur le Sida et les hépatites virales, Expertise France), a pour objectif le développement de plateformes polyvalentes ouvertes de diagnostic. Ce modèle de plateforme s'oppose aux systèmes fermés proposés aujourd'hui par les fabricants qui ne peuvent fonctionner qu'avec les réactifs provenant du fournisseur de la plateforme, créant une situation d'exclusivité n’offrant aucune possibilité de négociations les prix aux laboratoires d'analyse. Les plateformes polyvalentes ouvertes offrent une structure sur laquelle des éléments provenant de différents fournisseurs peuvent être combinés. L’objectif est de permettre l'ouverture du marché à la concurrence afin de baisser les prix des tests. Les performances de la plateforme développée dans le cadre du projet OPP-ERA, ainsi que celles de tests génériques de quantification de l'ADN du VHB et de l'ARN du VIH pouvant être utilisées sur la plupart des plateformes d'analyse actuellement commercialisées, ont récemment été validées [38].

Le développement d'outils en accès libre pourrait aussi avoir un rôle à jouer dans le changement de paradigme de la fabrication de tests diagnostiques. En effet, plusieurs plateformes de partage de protocole d'analyses biologiques et chimiques ont été mises en place pour faciliter l'accès à ces procédés ainsi que leur développement de manière collaborative [39]. Le développement de matériel informatique dont le fonctionnement est ouvert et complètement reprogrammable en fonction des besoins de l'utilisateur, comme les modules Arduino ou Raspberry Pi, ont permis d'étendre ce principe au partage de protocoles de création d'outils. Ainsi, un thermocycleur - appareil permettant le contrôle des variations de température nécessaires à l'amplification du matériel génétique - à bas coût a pu être développé et l'ensemble de ses plans mis en libre accès [40].

L'utilisation de ces outils pourrait donc s'inscrire dans une démarche de fabrication « maison » de tests diagnostiques, déjà présente dans certains PED-PMA. En effet, en raison des prix élevés des kits commerciaux, de nombreux laboratoires de ces pays disposant du savoir-faire nécessaire développent leurs propres techniques d'analyse « maison ». Ces protocoles moins chers s’adaptent aux outils et réactifs disponibles dans ces pays. Les machines nécessaires à la fabrication de certains outils présentés dans la section 2.1 sont maintenant accessibles au grand public, dans des formats compacts et à moindre coût. Par exemple, des imprimantes 3D ou d'impression en relief, comme celles requises pour l'impression de micro-fluides sur papier, à moins de 250 dollars sont maintenant disponibles.

La mise en place d'un tel système soulève bien sûr un certain nombre de questions, déjà présentes pour la plupart dans l'étude des innovations dans le cadre des PED-PMA, 
telles que l'impact de la propriété intellectuelle sur l'accès de ces pays aux innovations technologiques, la viabilité économique des systèmes reposant sur des technologies en libre accès ainsi que la nécessité de normes permettant de contrôler la qualité des tests diagnostiques distribués au vu des enjeux de santé et économiques en présence.

\section{Conclusion}

Une analyse de la littérature scientifique portant sur les innovations diagnostiques montre le foisonnement dont ce champ fait preuve. Une grande variété de technologies pourrait ainsi permettre le développement de tests diagnostiques rapides adaptés au contexte des PED-PMA, la disponibilité de tels tests étant considérée comme la pierre manquante pour garantir l'efficacité de la cascade de soins du VHC dans ces pays. Cependant, un manque de coordination et de partage de savoirs entre, d'une part, les différents secteurs impliqués dans la création de ces innovations et, d'autre part, ceux pouvant favoriser et autoriser leur adoption sur le terrain empêche le passage à la production de grande ampleur de ces innovations. Des pistes de réflexion et de contournement de ces difficultés sont à l'étude mais l'isolement de telles initiatives empêche leur mise en pratique. Le développement d'une approche croisée entre les disciplines techniques et les sciences sociales permettrait donc de développer un cadre de lecture plus précis de cette problématique et d'identifier des solutions permettant la mise en œuvre opérationnelle de ces outils. 


\section{Bibliographie}

[1] Stanaway JD, Flaxman AD, Naghavi M, Fitzmaurice C, Vos T, Abubakar I, et al. The global burden of viral hepatitis from 1990 to 2013: findings from the Global Burden of Disease Study 2013. Lancet. 2016 Sep 10;388(10049):1081-8.

[2] WHO. Global hepatitis report, 2017 [Internet]. WHO. [cited 2017 Aug 3]. Available from: http:/www.who.int/hepatitis/publications/global-hepatitisreport2017/en/

[3] WHO. Hepatitis C [Internet]. WHO. [cited 2018 Jul 18]. Available from: http://www.who.int/news-room/fact-sheets/detail/hepatitis-c/

[4] Gower E, Estes C, Blach S, Razavi-Shearer K, Razavi H. Global epidemiology and genotype distribution of the hepatitis C virus infection. J Hepatol. 2014 Nov;61(1 Suppl):S45-57.

[5] WHO. Progress report on access to hepatitis C treatment [Internet]. [cited 2018 Sep 26]. Available from: http://www.who.int/hepatitis/publications/hep-c-access-report2018/en/

[6] Grillon C, Krishtel PR, Mellouk O, Basenko A, Freeman J, Mendão L, et al. Treatment advocate tactics to expand access to antiviral therapy for HIV and viral hepatitis $\mathrm{C}$ in low- to high-income settings: making sure no one is left behind. $\mathrm{J}$ Int AIDS Soc. 2018 Apr;21 Suppl 2:e25060.

[7] Ishizaki A, Bouscaillou J, Luhmann N, Liu S, Chua R, Walsh N, et al. Survey of programmatic experiences and challenges in delivery of hepatitis $\mathrm{B}$ and $\mathrm{C}$ testing in low- and middle-income countries. BMC Infect Dis. 2017 01;17(Suppl 1):696.

[8] Kuehn BM. Global Shortage of Health Workers, Brain Drain Stress Developing Countries. JAMA. 2007 Oct 24;298(16):1853-5.

[9] Wade AJ, Veronese V, Hellard ME, Doyle JS. A systematic review of community based hepatitis C treatment. BMC Infect Dis [Internet]. 2016 May 16 [cited 2017 Dec 9];16. Available from: https://www.ncbi.nlm.nih.gov/pmc/articles/PMC4867528/

[10] WHO. Global policy report on the prevention and control of viral hepatitis [Internet]. WHO. [cited 2017 Sep 25]. Available from: http://www.who.int/hiv/pub/hepatitis/global_report/en/

[11] Shimakawa Y, Pourette D, Bainilago L, Enel C, Sombié R, Rado R, et al. Improving communication about viral hepatitis in Africa. Lancet Infect Dis. 2017 Jul;17(7):688-9.

[12] Freiman JM, Tran TM, Schumacher SG, White LF, Ongarello S, Cohn J, et al. Hepatitis C Core Antigen Testing for Diagnosis of Hepatitis C Virus Infection: A Systematic Review and Meta-analysis. Ann Intern Med. 2016 Jun 21; 
[13] Lange B, Roberts T, Cohn J, Greenman J, Camp J, Ishizaki A, et al. Diagnostic accuracy of detection and quantification of HBV-DNA and HCV-RNA using dried blood spot (DBS) samples - a systematic review and meta-analysis. BMC Infect Dis. 2017 01;17(Suppl 1):693.

[14] Soulier A, Poiteau L, Rosa I, Hézode C, Roudot-Thoraval F, Pawlotsky J-M, et al. Dried Blood Spots: A Tool to Ensure Broad Access to Hepatitis C Screening, Diagnosis, and Treatment Monitoring. J Infect Dis. 2016 Apr 1;213(7):1087-95.

[15] Gupta E, Agarwala P, Kumar G, Maiwall R, Sarin SK. Point -of -care testing (POCT) in molecular diagnostics: Performance evaluation of GeneXpert HCV RNA test in diagnosing and monitoring of HCV infection. J Clin Virol. 2017 Mar;88:46-51.

[16] Streets AM, Huang Y. Chip in a lab: Microfluidics for next generation life science research. Biomicrofluidics. 2013 Jan;7(1):11302.

[17] Choi JR, Tang R, Wang S, Wan Abas WAB, Pingguan-Murphy B, Xu F. Paperbased sample-to-answer molecular diagnostic platform for point-of-care diagnostics. Biosens Bioelectron. 2015 Dec 15;74:427-39.

[18] Mou L, Jiang X. Materials for Microfluidic Immunoassays: A Review. Adv Healthc Mater. 2017 Mar 21;

[19] Bhalla N, Jolly P, Formisano N, Estrela P. Introduction to biosensors. Essays Biochem. 2016 Jun 30;60(1):1-8.

[20] Slomovic S, Pardee K, Collins JJ. Synthetic biology devices for in vitro and in vivo diagnostics. Proc Natl Acad Sci USA. 2015 Nov 24;112(47):14429-35.

[21] Sharma S, Zapatero-Rodríguez J, Estrela P, O’Kennedy R. Point-of-Care Diagnostics in Low Resource Settings: Present Status and Future Role of Microfluidics. Biosensors (Basel). 2015 Aug 13;5(3):577-601.

[22] Breslauer DN, Maamari RN, Switz NA, Lam WA, Fletcher DA. Mobile Phone Based Clinical Microscopy for Global Health Applications. PLoS One [Internet]. 2009 Jul 22 [cited 2018 Sep 26];4(7). Available from: https://www.ncbi.nlm.nih.gov/pmc/articles/PMC2709430/

[23] Selck DA, Karymov MA, Sun B, Ismagilov RF. Increased Robustness of SingleMolecule Counting with Microfluidics, Digital Isothermal Amplification, and a Mobile Phone versus Real-Time Kinetic Measurements. Anal Chem. 2013 Nov 19;85(22):11129-36.

[24] Pardee K, Green AA, Takahashi MK, Braff D, Lambert G, Lee JW, et al. Rapid, Low-Cost Detection of Zika Virus Using Programmable Biomolecular Components. Cell. 2016 May 19;165(5):1255-66. 
[25] Wong AP, Gupta M, Shevkoplyas SS, Whitesides GM. Egg beater as centrifuge: isolating human blood plasma from whole blood in resource-poor settings. Lab Chip. 2008 Dec;8(12):2032-7.

[26] Brown J, Theis L, Kerr L, Zakhidova N, O’Connor K, Uthman M, et al. A HandPowered, Portable, Low-Cost Centrifuge for Diagnosing Anemia in Low-Resource Settings. Am J Trop Med Hyg. 2011 Aug 1;85(2):327-32.

[27] Bhamla MS, Benson B, Chai C, Katsikis G, Johri A, Prakash M. Hand-powered ultralow-cost paper centrifuge. Nature Biomedical Engineering. 2017 Jan;1(1):0009.

[28] Korir G, Prakash M. Punch card programmable microfluidics. PLoS ONE. 2015;10(3):e0115993.

[29] Bhatti YA, Ventresca M. How Can 'Frugal Innovation' Be Conceptualized? [Internet]. Rochester, NY: Social Science Research Network; 2013 Jan [cited 2018 Sep 26]. Report No.: ID 2203552. Available from: https://papers.ssrn.com/abstract=2203552

[30] Haudeville B, Bas CL. L'innovation frugale, paradigme technologique naissant ou nouveau modèle d'innovation ?, Frugal Innovation: Emerging Technological Paradigm or New Model of Innovation? Innovations. 2016 Sep 23;(51):9-25.

[31] Haudeville B. À propos de l'innovation frugale. Mondes en développement. 2016 Apr 5;(173):7-9.

[32] Prime M, Attaelmanan I, Imbuldeniya A, Harris M, Darzi A, Bhatti Y. From Malawi to Middlesex: the case of the Arbutus Drill Cover System as an example of the cost-saving potential of frugal innovations for the UK NHS. BMJ Innovations. 2018 Apr;4(2):103-10.

[33] Bhatti Y, Taylor A, Harris M, Wadge H, Escobar E, Prime M, et al. Global Lessons In Frugal Innovation To Improve Health Care Delivery In The United States. Health Aff (Millwood). 2017;36(11):1912-9.

[34] Sackmann EK, Fulton AL, Beebe DJ. The present and future role of microfluidics in biomedical research. Nature. 2014 Mar 13;507(7491):181-9.

[35] Becker H. It’s the economy.. Lab Chip. 2009 Oct 7;9(19):2759-62.

[36] McNerney R, Peeling RW. Regulatory In Vitro Diagnostics Landscape in Africa: Update on Regional Activities. Clin Infect Dis. 2015 Oct 15;61Suppl 3:S135-140.

[37] Rugera SP, McNerney R, Poon AK, Akimana G, Mariki RF, Kajumbula H, et al. Regulation of medical diagnostics and medical devices in the East African community partner states. BMC Health Serv Res. 2014 Oct 31;14:524.

[38] Kania D, Rouet F, Bollore K, Nouhin J, Njouom R, Toni Td’A, Maiga A, ToureKane C, Ngo-Giang-Huong N, Dagnra A, Chuong Le DH, Lunel-Fabiani F, Chaix M-L, Castera-Guy J, Da Costa Castro J-M, Rouzioux C, Plantier J-C, Tuaillon E. Validation and scale-up of Hepatitis B viral load on polyvalent open PCR platforms in West 
African and South East Asian countries. A study of the AC12 working group (ANRS 12327). Poster TUPEB0391 Exhibition at: 9th IAS Conference on HIV Science (IAS 2017); 2017 Jul 23-26; Paris, France.

[39] Open-source biology. Nature. 2004 Sep;431(7008):491.

[40] BHA. Thermocycler: Thermocycler for PCR [Internet]. BioHack Academy; 2017 [cited 2017 Jun 24]. Available from: https://github.com/BioHackAcademy/BHA_Thermocycler 